\title{
Molecular cloning of the small $(\gamma)$ subunit of human TFIIA reveals functions critical for activated transcription
}

\author{
Josef Ozer, ${ }^{1,3}$ Paul A. Moore, ${ }^{2,3}$ Arthur H. Bolden, ${ }^{1}$ Arianna Lee, ${ }^{1}$ Craig A. Rosen, ${ }^{2}$ and \\ Paul M. Lieberman ${ }^{1,4}$ \\ ${ }^{1}$ Roche Institute of Molecular Biology, Nutley, New Jersey 07110-1199 USA; ${ }^{2}$ Human Genome Sciences, Inc., Rockville, \\ Maryland 20850-3338 USA
}

\begin{abstract}
TFIIA is thought to play an important role in transcriptional regulation in higher eukaryotes, but its precise function is unclear. A human cDNA encoding a protein with $45 \%$ identity to the small subunit of yeast TFIIA has been isolated. TFIIA activity could be reconstituted by the mixing of recombinant large ( $\alpha \beta)$ and small $(\gamma)$ subunits. TFIIA-depleted HeLa nuclear extracts were used to demonstrate that TFIIA is essential for basal and activated transcription by several distinct classes of activators. Recombinant TFIIA functioned in transcriptional activation whether expressed as a dimer $(\alpha \beta+\gamma)$ or as a trimer $(\alpha+\beta+\gamma)$, which closely resembles the native form. Yeast TFIIA also functioned in transcriptional activation, and the human $\gamma$ subunit was functionally interchangeable with TOA2, its yeast homolog. Recombinant TFIIA mediated the stimulation of TFIID binding to the TATA region and downstream promoter sequences by the Zta transcriptional activator. Significantly, we found that TFIIA bound directly to $\mathrm{Zta}$ in an activation domain-dependent manner. One consequence of the TFIIA-mediated interaction between Zta and TFIID was the formation of a promoter-bound complex resistant to TATA oligonucleotide competition. These results demonstrate that TFIIA is an evolutionarily conserved general factor critical for activator-regulated transcription.
\end{abstract}

[Key Words: Transcriptional activation; TFIIA; EST cloning; coactivator]

Received August 5, 1994; revised version accepted August 30, 1994

Eukaryotic transcriptional activators modulate the formation of a preinitiation complex composed of general transcription factors and RNA polymerase II (for review, see Tjian and Maniatis 1994). The general transcription factors (GTFs) TFIIA, TFIIB, TFIID, TFIIE, TFIIF, TFIIG/ $\mathrm{J}$, and TFIIH have been shown to stimulate correct initiation by RNA polymerase II in cell-free transcription reactions (for review, see Conaway and Conaway 1993; Zawel and Reinberg 1993). More recently, a minimal transcription system has been reconstituted with only the TATA-binding protein (TBP) core of TFIID, TFIIB, the small subunit of TFIIF (RAP30), and RNA polymerase II (Tyree et al. 1993). However, this minimal transcription system cannot be regulated by promoter-specific activators. The components required for activated transcription appear to be much more complex than those necessary for the minimal reactions, and several activities, referred to as coactivators or mediators, have been shown to participate in regulated transcription in addition to the full set of general transcription factors

${ }^{3}$ These authors contributed equally to this work. ${ }^{4}$ Corresponding author.
(Meisterernst et al. 1991; Kim et al. 1994; Koleske and Young 1994; for review, see Gill and Tjian 1992; Drapkin et al. 1993).

In higher eukaryotes, TFIID consists of TBP and a set of TBP-associated factors (TAFs) that are essential for activated transcription (Dynlacht et al. 1991; Tanese et al. 1991; Pugh and Tjian 1992; Zhou et al. 1992). TAFs have been shown to interact with transcriptional activators (Goodrich et al. 1993; Hoey et al. 1993; Wienzierl et al. 1993), with other GTFs (Goodrich et al. 1993; Yokomori et al. 1993), and with promoter sequences overlapping the transcriptional initiation site (Kaufman and Smale 1994; Purnell et al. 1994; Verrijzer et al. 1994). Like the TAFs, TFIIA is not required in basal transcription systems reconstituted just with the TBP component of TFIID (Cortes et al. 1992; Tyree et al. 1993). However, TFIIA stimulates basal levels in reactions reconstituted with TFIID (where TBP is associated with TAFs) in addition to the required, but partially purified, set of the remaining mammalian GTFs (Cortes et al. 1992; Conaway and Conaway 1993; Zawel and Reinberg 1993). Similarly, to the TAFs, TFIIA binds directly to TBP and copurifies with it chromatographically over several columns, including the immunopurification of TBP under 
certain conditions (Cortes et al. 1992; Ma et al. 1993; Yokomori et al. 1993).

The precise role of the GTF TFIIA in regulating transcription remains unclear. TFIIA is thought to be required for early steps in the assembly of an active preinitiation complex (Buratowski et al. 1989; Maldonado et al. 1990; Wang et al. 1992; Lieberman and Berk 1994). TFIIA binds directly to TBP and stabilizes the interaction of TBP with the TATA box in electrophoresis mobility shift assays (EMSA) (Buratowski et al. 1989; Maldonado et al. 1990; Lee et al. 1992; Imbalzano et al. 1994). The binding of TFIIA to TBP alters the DNA-binding properties of TBP and is likely to contribute to changes in promoter recognition and transcriptional activity (Lee et al. 1992). TFIIA was also required during a preincubation of TFIID with activator to overcome a rate-limiting step in open complex formation /Wang et al. 1992, Chi and Carey 1993). These latter experiments were also consistent with a model postulating TFIIA functions as a transcriptional derepressor by precluding transcriptional inhibitors from binding TFIID (Roeder 1991; Cortes et al. 1992). Several candidate inhibitors of TFIID have been identified by virtue of their direct interaction with TBP (Meisterernst and Roeder 1991; Inostroza et al. 1992; Auble and Hahn 1993; Merino et al. 1993).

In addition to functioning as a derepressor, TFIIA may also contribute directly to a true activation process. Evidence that TFIIA participates in such a manner has been presented in experiments with the Zta transcriptional activator (Lieberman and Berk 1994). Zta can stimulate the binding of TFIID to the TATA element and downstream sequences in a manner that is strongly dependent on TFIIA. This stimulation of promoter binding was strictly dependent on the TAFs and the Zta activation domain, further supporting the biological significance of this function (Lieberman and Berk 1994). However, these functional studies were performed with partially purified TFIIA; thus, TFIIA function could be attributed to substoichiometric quantities of coactivator or mediator contaminants. Confirmation of these conclusions requires the availability of either homogeneous or a recombinant form of TFIIA.

Human TFIIA has been purified as three polypeptides $\langle\alpha, \beta, \gamma\rangle$ with molecular masses of 35,19 , and $12 \mathrm{kD}$, respectively (DeJong and Roeder 1993; Ma et al. 1993). A yeast TFIIA activity has also been identified and consists of two polypeptides (32 and $13.5 \mathrm{kD}$ ) (Hahn et al. 1989; Ranish and Hahn 1991). The genes encoding these two subunits of yeast TFIIA, TOA1 and TOA2, respectively, have been shown to be essential for cell viability (Ranish et al. 1992). Both human and yeast TFIIA bind to the evolutionarily conserved carboxy-terminal domain of TBP (Buratowski and Zhou 1992) and can stimulate transcription in crude heterologous systems (Ranish and Hahn 1991; Ranish et al. 1992). Recently, the isolation of cDNAs encoding the large subunit of human and Drosophila TFIIA revealed that the $35-$ and $19-\mathrm{kD}$ subunits are derived from a single gene (referred to here as $\alpha \beta$ ). These $\alpha \beta$ subunits share sequence similarity with the large subunit of yeast TFIIA, encoded by TOAl (DeJong and Roeder 1993; Ma et al. 1993; Yokomori et al. 1993). Both the human and Drosophila TFIIA large subunit appear to be post-translationally proteolyzed into two smaller polypeptides of 35 and $19 \mathrm{kD}$ (referred to here as $\alpha$ and $\beta$, respectively).

Isolation of the TFIIA large subunit has allowed some functional questions to be addressed. The unproteolyzed recombinant large subunit of human TFIIA could be combined with gel-purified human small subunit (p12) to generate TBP binding in EMSAs (DeJong and Roeder 1993). Antibodies derived against the large subunit of TFIIA supershifted TBP-TFIIA complexes and specifically inhibited activated transcription, but did not interfere with TBP-directed basal level transcription (Ma et al. 1993). To better characterize the role of TFIIA in the transcriptional activation process, we have isolated the gene encoding the small subunit of human TFIIA $(\gamma)$. The availability of the small subunit has now allowed us to test the importance of highly purified recombinant TFIIA in the regulation of RNA polymerase II transcription.

\section{Results}

Isolation of a cDNA encoding the human $\gamma$ TFIIA subunit

Previously, we have observed that yeast TFIIA could functionally substitute for human TFIIA in several biochemical assays important for transcriptional activation (data not shown). Thus, it appeared likely that yeast and human TFIIA would share sequence similarity. To identify a human gene encoding a protein with sequence similarity to yeast TOA2, a data base of expressed sequence tags (Adams et al. 1991, 1992) generated from multiple human tissues was searched using the BLAST algorithim (Altschul et al. 1990). A tag with homology to TOA2 was identified. This tag was used to again search the data base by BLAST analysis for a full-length cDNA. A fulllength clone containing an open reading frame of 109 amino acids was identified and isolated (Fig. 1A,B). Analysis of this human cDNA revealed that it encodes a protein that has $45 \%$ identical residues to yeast TOA2 $156 \%$ similarity including conservative substitutions). Further scrutiny of this data base revealed no evidence of multiple or closely related human genes.

To determine whether the human TOA2 cDNA homolog encoded a protein with TFIIA activity, we expressed both the human large $(\alpha \beta)$ subunit and the putative small subunit $\langle\gamma|$ in Escherichia coli. Both subunits were tagged at the amino terminus to six histidine residues and purified to near homogeneity by affinity chromatography on Ni-NTA-agarose. TFIIA activity was first assayed for the stabilization of human recombinant TBP (hu-rTBP) binding to a TATA box-containing probe by EMSA analysis (Fig. 2A). We have found that hu-rTBP does not bind to promoter DNA stably in the absence of TFIIA in EMSA conducted in Mg-agarose gels (Fig. 2A, lane 2). Addition of $0.5 \mu \mathrm{g}$ of partially purified human 
A

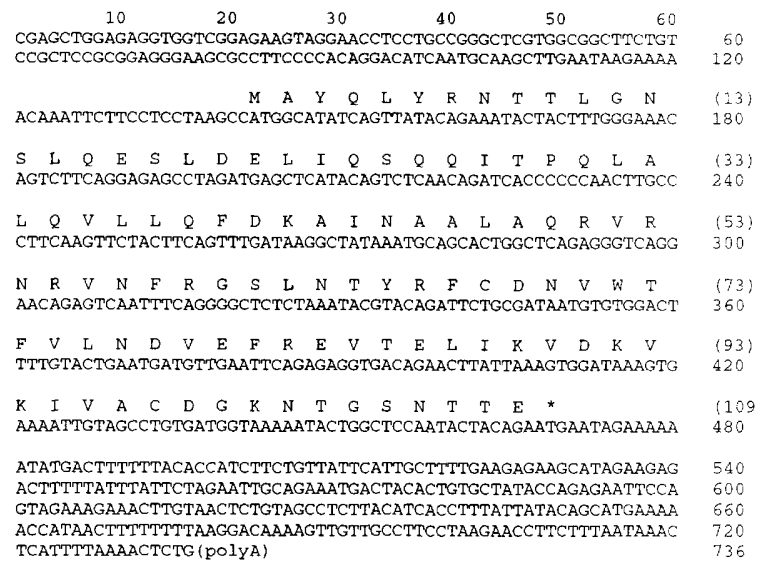
B

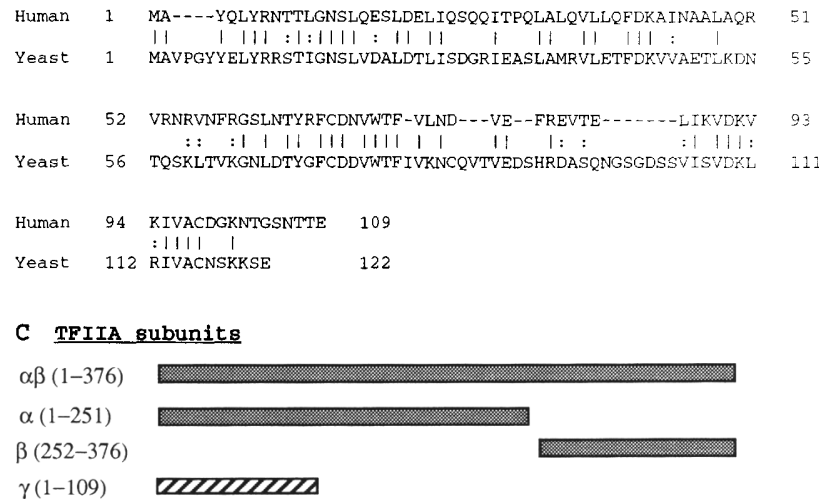

Figure 1. (A) Sequence of a cDNA encoding the small $|\gamma|$ subunit of hIIA. The molecular mass of the deduced 109-residue protein is 12,457 daltons, with a calculated pI of 6.55 . (B) Comparison of the amino acid composition of human and yeast TFIIA small subunits. The human TFIIA- $\gamma$ subunit is $45 \%$ identical to yeast TOA2, with $56 \%$ similarity overall. $(C)$ Schematic diagram depicting the hIIA subunits. TFIIA is encoded by two genes, $\alpha \beta$ and $\gamma$. The $\alpha \beta$ protein is processed post-translationally into two polypeptides $(\alpha$ and $\beta),-35$ and $19 \mathrm{kD}$, respectively. Recombinant $\alpha$ and $\beta$ polypeptides were designed with a break point at amino acid residue 251 , but this may not exactly correspond to the naturally occurring proteolytic cleavage site, which is not yet reported.

TFIIA (hIIA) [DE-IIA fraction (Cortes et al. 1992)] to $10 \mathrm{ng}$ $(\sim 20 \mathrm{nM}$ final) of hu-rTBP resulted in the formation of a stable TBP-TFIIA DNA complex, referred to as D-A (lane 3). Addition of $0.2 \mu \mathrm{M}$ (final concentration) $\alpha \beta$ or $\gamma$ to hu-rTBP did not produce the D-A complex (lanes 4,5 ). However, the addition of $0.2 \mu \mathrm{M}$ of $\alpha \beta+\gamma$ to hu-rTBP resulted in the formation of a D-A complex with promoter DNA (lane 6). Recombinant $\alpha \beta+\gamma$ did not have any DNA-binding activity in the absence of hu-rTBP (lane 8 ). We also show that recombinant yeast TFIIA (y $\alpha \beta / y \gamma$ ) produces a D-A complex with hu-rTBP that has a slightly faster mobility than the hIIA-formed complexes (lane 7). This difference in mobility suggests that TFIIA remains bound to the TBP-DNA complex and may influence the overall conformation of the proteinDNA complex.

Because partially purified TFIIA stimulates basal transcription reactions that utilize TFIID, recombinant hIIA was tested for its ability to stimulate transcription from a strong basal promoter using a HeLa cell nuclear extract (Fig. 2B). A TFIIA-dependent in vitro transcription system was derived by passing the HeLa cell nuclear extract over Ni-NTA-agarose. Ni-NTA binds specifically to the seven tandem histidine residues in the large subunit of TFIIA and has been used to affinity-purify functional hIIA (DeJong et al. 1993; Ma et al. 1993). TFIIA-depleted nuclear extracts were found to lose the ability to produce a specifically initiated transcript from a strong basal promoter composed of the adenovirus major late promoter TATA box and the TdT initiator element (Smale and Baltimore 1989; Fig. 2B, cf. lanes 1 and 2). Addition of $\sim 2$
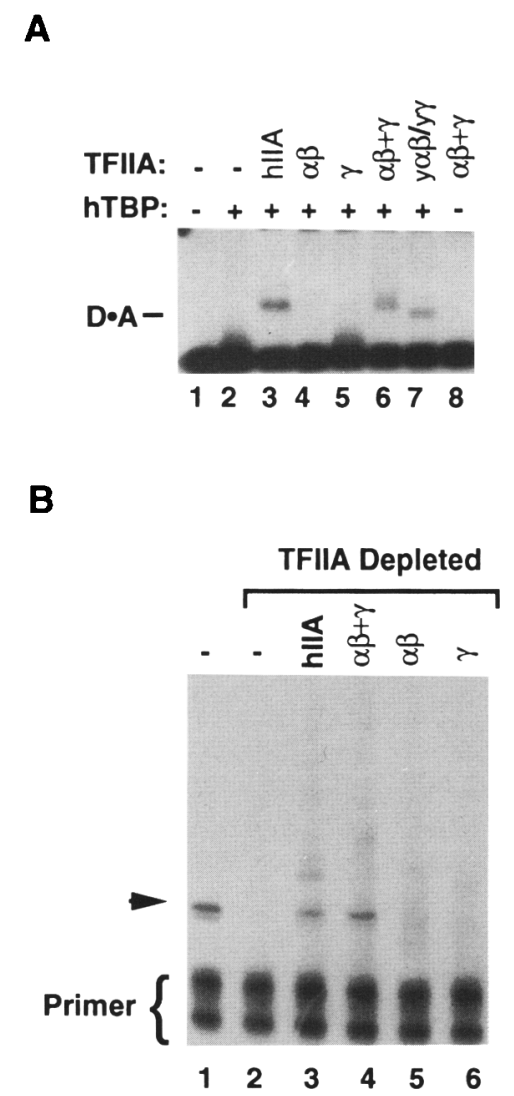

Figure 2. Recombinant TFIIA binds human TBP and stimulates basal transcription in TFIIA-depleted HeLa nuclear extracts. (A) Various TFIIA sources form a D-A complex as measured by $\mathrm{Mg}$-agarose EMSA. TBP (10 $\mathrm{ng})$ and radiolabeled $\mathrm{Z}_{7} \mathrm{E} 4 \mathrm{TCAT}$ promoter region $(-170$ to +54$)$ were incubated for $45 \mathrm{~min}$ at $30^{\circ} \mathrm{C}$ with $0.6 \mu \mathrm{g}$ of hIIA (lane 3) or $\sim 0.2 \mu \mathrm{m}$ final concentration of $\alpha \beta$ (lane 4), $\gamma$ (lane 5), $\alpha \beta+\gamma$ (lane 6), or yeast $\alpha \beta+\gamma$ (lane 7). $(B)$ In vitro transcription reactions with the TATA/Inr SP6 promoter in complete nuclear extracts $(40 \mu \mathrm{g}$; lane 1) or in TFIIA-depleted nuclear extracts $(40 \mu \mathrm{g}$; lanes 2-6). TFIIA-depleted nuclear extracts were supplemented with $2.4 \mu \mathrm{g}$ hIIA (lane 3) or $0.2 \mu \mathrm{M}$ final concentration of $\alpha \beta+\gamma$ (lane 4), $\alpha \beta$ (lane 5), or $\gamma$ (lane 6). Correctly initiated primer extension products are indicated by the arrow. 
$\mu g$ of partially purified hIIA (Cortes et al. 1992) to depleted extracts restored transcription to similar levels observed in the untreated extracts (lane 3). Addition of $0.2 \mu \mathrm{M} \alpha \beta$ did not restore transcription (lane 5), nor did addition of $0.2 \mu \mathrm{M}$ of $\gamma$ (lane 6). However, addition of 0.2 $\mu \mathrm{M} \alpha \beta+\gamma$ did restore transcription to levels comparable with that produced with $2 \mu \mathrm{g}$ of hIIA (lane 4). Concentrations of $\alpha \beta+\gamma$ as low as $0.075 \mu \mathrm{M}$ stimulated basal levels fivefold over levels detected in depleted extracts (data not shown). This result indicates that recombinant $\alpha \beta+\gamma$ has TFIIA transcriptional activity and that depletion of the $\alpha \beta$-subunit from HeLa cells results in a near stoichiometric depletion of the $\gamma$-subunit.

\section{TFIIA is required for transcription activated by several distinct activation domains}

TFIIA-depleted nuclear extracts were tested for their ability to support transcription stimulated by several distinct types of activators (Fig. 3). Three different classes of activation domains were fused to the GAL4 DNA-binding domain and examined for their ability to stimulate transcription from the $\mathrm{G}_{5} \mathrm{E} 1 \mathrm{BTCAT}$ promoter, which contains five GAL4-binding sites upstream of the adenovirus E1B TATA element. In untreated nuclear extracts the $\mathrm{G}_{5}$ E1BTCAT promoter produced low basal levels of transcription (Fig. 3A, lanes 1, top three panels). GAL4 fusions to the synthetic acidic activation domain (GAL4-AH), the herpes simplex virus-encoded VP16 activation domain (GAL4-VP16), or the proline-rich activation domain derived from the CCAAT-binding factor (GAL4-CTF) all stimulated transcription when fused to the GAL4 DNA-binding domain (lane 2). However, in TFIIA-depleted nuclear extracts, activated transcription was reduced significantly for all activators (lanes 3,4 ). Addition of $0.2 \mu \mathrm{M}$ recombinant $\alpha \beta+\gamma$ to the depleted extracts restored activated transcription to levels similar to that observed in the undepleted extracts (lanes 5,6 ). Addition of either the $\alpha \beta$ or $\gamma$ subunit alone $(0.2 \mu \mathrm{M})$ did not have any effect on restoring transcription by these activators (lanes 7,8; data not shown for Zta and GAL4$\mathrm{CTF}$ ). Addition of partially purified TFIIA (DE-IIA) resulted in similar levels of transcription as did $\alpha \beta+\gamma$ (lanes 9,10). A similar TFIIA dependence was observed for the Epstein-Barr virus-encoded $Z$ ta transcriptional activator with the $Z_{7} E 4 T C A T$ promoter, which contains seven $\mathrm{Zta}$-binding sites upstream of the adenovirus E4 TATA region (Fig. 3A, bottom). On average, Zta activated transcription between 8 - and 15 -fold over basal levels in the presence $0.2 \mu \mathrm{M}$ recombinant TFIIA in depleted extracts. In HeLa extracts partially depleted of TFIIA where basal levels could be measured, we found that $\mathrm{Zta}$ stimulated transcription only two- to threefold over basal (data not shown). This indicates that limiting concentrations of TFIIA significantly impair activated transcription.

One advantage of specifically depleting TFIIA activity from crude nuclear extracts is the ability to examine endogenous transcriptional activators. AP-1-mediated transcription was tested for its dependence on TFIIA by examining the activity of the collagenase promoter (Fig. 3B). Endogenous AP-1 activity was measured by comparing the wild-type collagenase promoter (AP-1) to a collagenase promoter that has the single AP-1 site deleted (mt). The AP-1 promoter produced high levels of transcription while the $\mathrm{mt}$ promoter was undetectable in untreated nuclear extracts (Fig. 3B, cf. lanes 1 and 4). In TFIIA-depleted nuclear extracts, AP-1-stimulated tran-
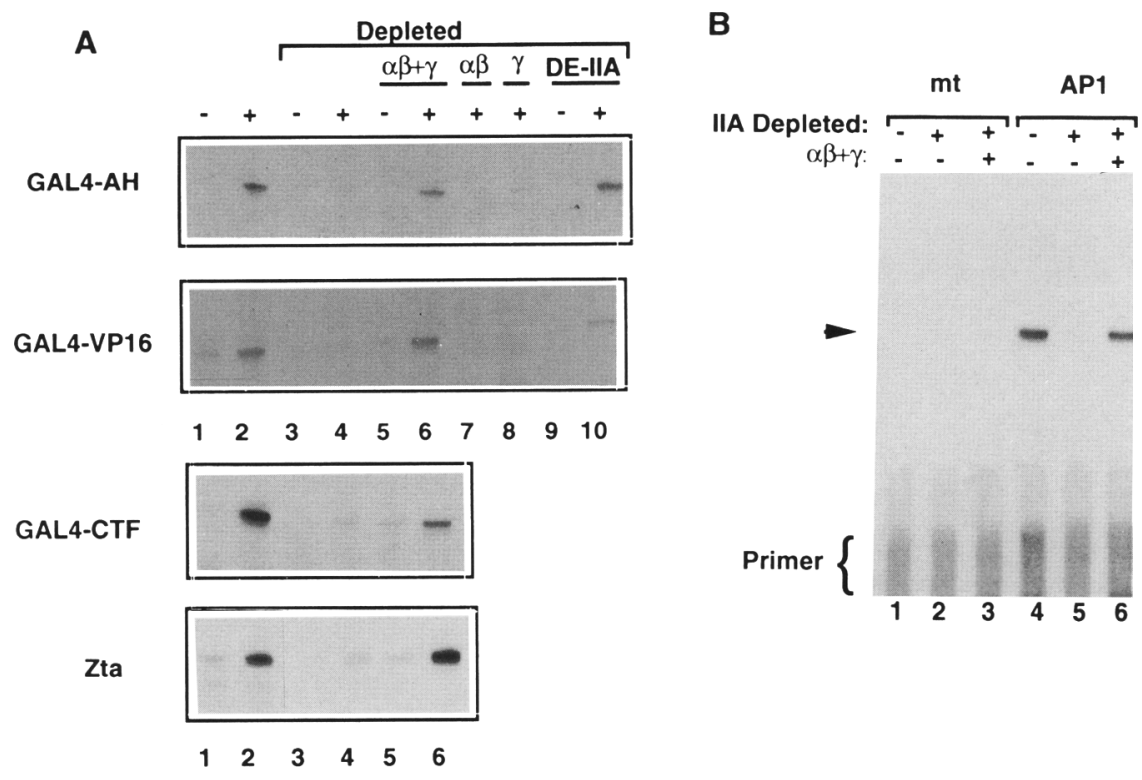

Figure 3. (A) Recombinant TFIIA restores activated transcription in TFIIA-depleted nuclear extracts. The activator proteins GAL4-AH, GAL4-VP16, GAL4-CTF, and Zta were incubated as indicated $(+\mid$ with untreated HeLa cell nuclear extract $(40 \mu \mathrm{g}$; lanes 1,2) or with TFIIA-depleted HeLa cell nuclear extracts $(40 \mu \mathrm{g}$; lanes $3-10)$ in in vitro transcription reactions. A final concentration of $0.2 \mu \mathrm{M}$ recombinant $\alpha \beta+\gamma$ (lanes 5,6), $\alpha \beta$ (lane 7), $\gamma$ (lane 8), or $2.4 \mu \mathrm{g}$ of partially purified hIIA (DE-IIA) (lanes $9,10)$ was supplemented to depleted extracts. Activators for primer extension products are indicated to the left of each panel. (B) Endogenous AP-1 activity requires TFIIA function. Untreated nuclear extract $(40 \mu \mathrm{g}$, lanes 1,4$)$ or TFIIA-depleted nuclear extract $(40 \mu \mathrm{g}$; lanes $2,3,5,6)$ were incubated with wild-type collagenase promoter $(-75$ to +63$)(\mathrm{AP}-1$; lanes $4-6)$ or an AP-1 deletion mutant of the collagenase promoter $(-60$ to +63$)\left(\mathrm{mt}_{\text {; }}\right.$ lanes $\left.1-3\right)$. Recombinant $\alpha \beta+\gamma$ was added to a final concentration of $0.2 \mu \mathrm{M}$ (lanes 3,6). Correctly initiated primer extension products are indicated by the arrow. 
scription was undetectable (lane 5). Addition of recombinant $\alpha \beta+\gamma(0.2 \mu \mathrm{M})$ restored transcription levels to those observed in the untreated extracts (lane 6). Importantly, addition of $\alpha \beta+\gamma$ did not stimulate the mt promoter (lane 3), indicating that TFIIA-stimulated levels were strictly dependent on the AP-1 activator.

We have shown that several distinct activators cannot function in the TFIIA-depleted Hela cell nuclear extracts and that adding back recombinant $\alpha \beta+\gamma$ rescued activator function. Next, we tested the ability of recombinant TFIIA to substitute for partially purified TFIIA in a transcription assay reconstituted with immunoaffinity-purified TFIID, recombinant TFIIB, partially purified RNA polymerase II/TFIIF/TFIIE, the upstream stimulating activity (USA) coactivator fraction, and recombinant activator (Fig. 4). In reconstituted reactions lacking any source of TFIIA, no transcription was observed with or without the GAL4-AH activator from the $\mathrm{G}_{5} \mathrm{E} 1 \mathrm{BTCAT}$ promoter (Fig. 4A, lanes 1,2). Addition of $\alpha \beta+\gamma(0.2 \mu \mathrm{M})$ produced high levels of transcription in the presence of GAL4-AH and no detectable basal levels (lanes 3 and 4). Addition of $2 \mu \mathrm{g}$ of partially purified TFIIA produced similar levels of transcription in the GAL4-AH-activated reactions and no detectable basal levels (lanes 5,6). A similar experiment was conducted using the $\mathrm{Z}_{7} \mathrm{E} 4 \mathrm{TCAT}$ promoter and the $\mathrm{Zta}$ activator (Fig. 4B). The results were similar to GAL4-AH, indicating that even in reconstituted transcription reactions with highly purified TFIID and TFIIB, and partially purified remaining factors, TFIIA activity is essential for transcriptional activation and can be completely restored with the recombinant $\alpha \beta$ and $\gamma$ polypeptides.

\section{TFIIA can function as a heterodimer or heterotrimer}

In HeLa cells and Drosophila embryo extracts, TFIIA activity is comprised of three polypeptides $\langle\alpha, \beta$, and $\gamma|$, which are approximately 35,19 , and $12 \mathrm{kD}$ in size. The two large polypeptides are derived from a single gene that encodes the $\alpha \beta$ protein. In contrast, yeast TFIIA can be isolated as two polypeptides (referred to here as $\mathrm{y} \alpha \beta$ and $y \gamma$ ) of 32 and $13.5 \mathrm{kD}$. To determine whether there were significant differences in the activity of the dimeric and the trimeric forms of TFIIA, the human $\alpha \beta, \alpha, \beta$, and $\gamma$, and the yeast $\alpha \beta$ and $\gamma$ polypeptides were all expressed and purified from E. coli. Various forms of TFIIA were examined for their ability to form the D-A complex in EMSA with $10 \mathrm{ng}$ of recombinant yeast TBP (YTBP) and a 29-bp oligonucleotide containing the adenovirus E1B TATA element (Fig. 5A). Under typical EMSA conditions in polyacrylamide gels, yTBP fails to bind DNA in the absence of TFIIA (Fig. 5A, lane 2). Addition of partially purified TFIIA (hIIA) produced a strong D-A complex in the presence of YTBP (lane 4) and had no DNAbinding activity in the absence of yTBP (lane 3). Addition of $\alpha \beta+\gamma$ to yTBP resulted in a D-A complex that resolved during electrophoresis as a triplet with mobility different from that of hIIA (cf. lanes 6 and 4). Addition of $\alpha \beta$ to yTBP had no effect on DNA binding (lane 8), nor did the addition of $\gamma$ alone (lane 10). The combination of $\alpha+\gamma$ also had no effect on yTBP binding (lane 12). Interestingly, we observed that the addition of $\beta+\gamma$ weakly stimulated yTBP binding yet produced a D-A complex with significantly faster mobility than hIIA or $\alpha \beta+\gamma$ (lane 14). The combination of $\alpha+\beta+\gamma$ resulted in a strong stimulation of yTBP binding and produced a D-A complex with mobility similar to that of native hIIA (lane 16). As was observed with human TBP in $\mathrm{Mg}$-agarose gel EMSA (Fig. $2 \mathrm{~A})$, yeast $\alpha \beta+\gamma(\mathrm{y} \alpha \beta / \mathrm{y} \gamma)$ strongly stimulated yTBP binding and produced a D-A complex with mobility faster than hIIA (lane 18). We also tested whether the human $\alpha \beta$ subunit could heterodimerize with the yeast $\gamma$ subunit $(h \alpha \beta / y \gamma)$, which produced a strong D-A complex triplet (lane 20). These results suggest that although the $\gamma$ - and $\beta$-subunits may be critical in mediating the binding of the TBP-DNA complex (lane 14), all three subunits contribute to the stable formation of the D-A complex. In addition, the human $\alpha \beta$ polypeptide is associated with the formation of D-A complex multimers (lanes 6,20). These results also indicate the
Figure 4. Requirement of TFIIA in transcription reactions reconstituted with holo-IID and partially purified HeLa factors. (A) The GAL4-AH transcriptional activator protein (175 ng) (lanes $2,4,6)$ was added to reactions containing $5 \mu \mathrm{l}$ of hIID, $100 \mathrm{ng}$ of $\mathrm{r}$-TFIIB, $5 \mu \mathrm{g}$ of RNA polymerase II, E/F fraction, $0.5 \mu \mathrm{g}$ of USA, G ${ }_{5}$ E1BTCAT template, and no TFIIA (lanes 1,2), $0.2 \mu \mathrm{M} \alpha \beta+\gamma$ (lanes 3,4 ), or $2.4 \mu \mathrm{g}$ of hIIA (lanes 5,6 ). (B) The same as in $A$ except that $150 \mathrm{ng}$ of $Z$ ta was added (lanes $2,4,6$ ) with the $Z_{7}$ E4TCAT template.

\section{A}

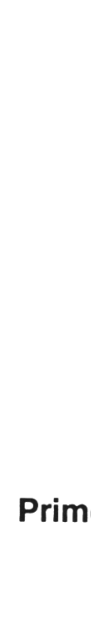

GAL4-AH

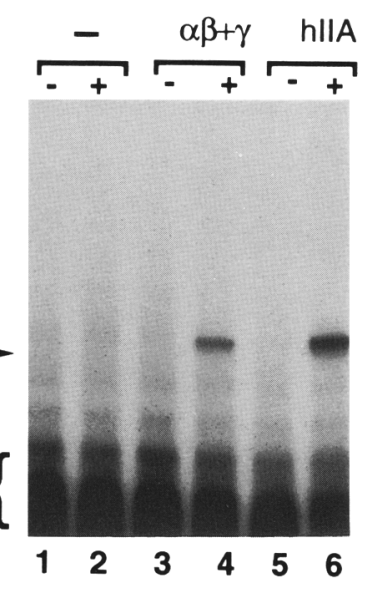

B

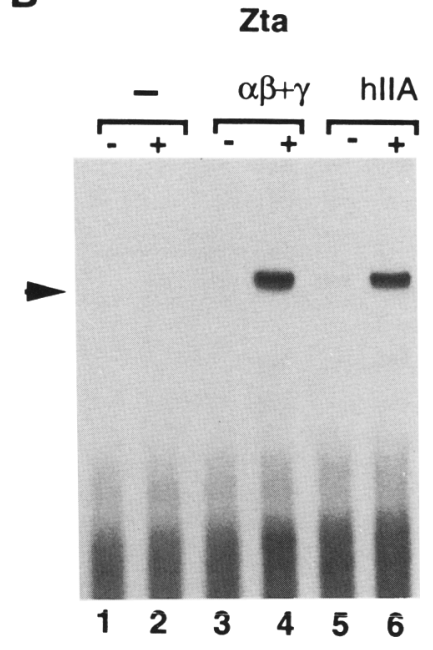


A

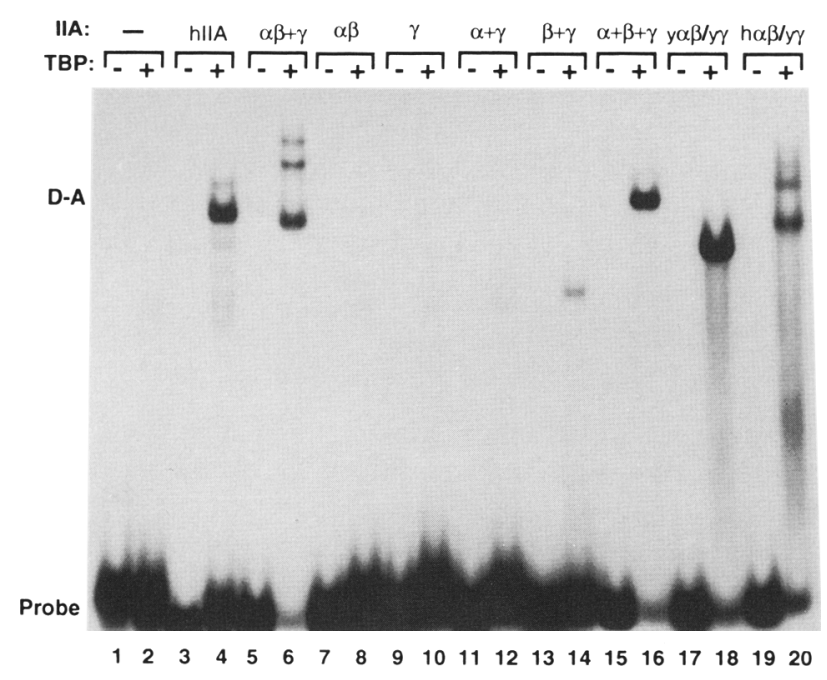

B

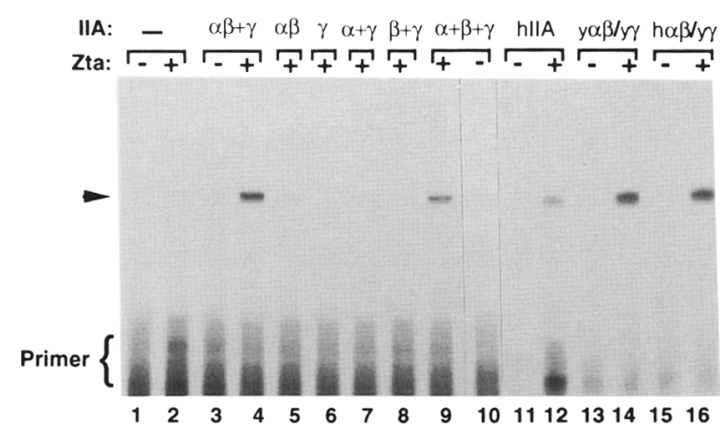

Figure 5. Functional activity of recombinant human, yeast, and heterologous TFIIA. $|A|$ Formation of a D-A complex bound to DNA detected by polyacrylamide gel EMSA. A 29-bp oligonucleotide probe containing the adenovirus E1B TATA element was incubated with various preparations of TFIIA in the absence $(-)$ or presence $(+)$ of $10 \mathrm{ng}$ of yTBP as indicated above each lane. Approximately $0.2 \mu \mathrm{M}$ final concentration of recombinant TFIIA was incubated in each reaction. Partially purified hIIA (lanes 3,4 ), combinations of recombinant human subunits $\{\alpha \beta$, $\alpha, \beta, \gamma$, lanes $5-16)$, recombinant yeast $\alpha \beta+\gamma(\mathrm{y} \alpha \beta / \mathrm{y} \gamma$, lanes 17,18 ) or a mixture of the human $\alpha \beta$ with yeast $\gamma$ (h $\alpha \beta / y \gamma$, lanes $19,20)$ are indicated above each lane. $(B)$ Ability of various TFIIA proteins to function in transcriptional activation by Zta. Transcription reactions were reconstituted as in Fig. 4 with immunoaffinity-purified TFIID (hIID), recombinant TFIIB, partially purified RNA polymerase II/IIE/IIF, and USA with (+) or without $(-\mid \mathrm{Zta}$. Various TFIIA preparations were added to reactions as indicated above each lane. The arrow indicates the correctly initiated transcript.

interaction between $\alpha \beta$ and $\gamma$ is evolutionarily conserved between human and yeast.

The various TFIIA species were tested for their ability to support transcriptional activation by the Zta activator in a reconstituted transcription reaction similar to that described for Figure 4 (Fig. 5B). As observed previously, human $\alpha \beta+\gamma$ supported strong activation by Zta (lane 4). The $\alpha \beta, \gamma, \alpha+\gamma$, and $\beta+\gamma$ polypeptide combinations did not support activated transcription (lanes 5-8). The het- erotrimeric $\alpha+\beta+\gamma$ species activated transcription in the presence of Zta (lane 9) but did not stimulate detectable levels of basal transcription (lane 10), similar to $\alpha \beta+\gamma$. In this assay hIIA also supported Zta activation, although at slightly lower levels than the recombinant protein (lane 12). Interestingly, the yeast $\alpha \beta+\gamma$ and the human/yeast heterodimer $(\mathrm{h} \alpha \beta / \mathrm{y} \gamma)$ also supported high levels of Zta transcriptional activation (lanes 14 and 16, respectively|. In conclusion, TFIIA species that formed a stable D-A complex were also capable of supporting transcriptional activation by $\mathrm{Zta}$. These results clearly indicate that the dimeric and trimeric forms of TFIIA are functionally equivalent for transcriptional activation in vitro and that this TFIIA function is evolutionarily conserved between yeast and human.

\section{Recombinant TFIIA mediates an activator-dependent stimulation of TFID-promoter binding}

The mechanistic function of TFIIA in the activation process remains to be fully elucidated. Previously, we observed that partially purified TFIIA is required for an activation domain-dependent stimulation of TFIID binding to promoter DNA (Lieberman and Berk 1994). We now demonstrate that highly purified recombinant TFIIA behaves identically to the partially purified IIA in this function (Fig. 6). Promoter-DNA binding reactions with limiting amounts of immunoaffinity-purified TFIID (hIID) were assayed by DNase I protection (Fig. $6 \mathrm{~A})$. Addition of $10 \mu \mathrm{l}$ of hIID resulted in barely detectable protection of the TATA element (Fig. 6A, lane 2). The combination of hIID and recombinant $\alpha \beta+\gamma(0.2$ $\mu M)$ had a small stimulatory effect on hIID binding to the promoter TATA region (lane 3). Addition of $\alpha \beta+\gamma$ by itself had no detectable binding activity (lane 4). The Zta tanscriptional activator was added at concentrations just below saturation $(20 \mathrm{nM})$ and weakly protected the ZRE sites (lane 5). Addition of hIID in the presence of $\mathrm{Zta}$ resulted in weak protection of the TATA sequence (lane 6). However, addition of hIID in the presence of $\mathrm{Zta}$ and $\alpha \beta+\gamma$ resulted in a large stimulation of hIID binding that completely protected the TATA region from -40 to -16 (lane 7). In the presence of Zta, $\alpha \beta+\gamma$, and hIID, promoter sequences downstream from the TATA region were also protected from +12 to +28 , and DNase I hypersensitive sites were induced at positions +2 and +12 . Furthermore, the Zta-binding sites were better protected (lane 7), indicating that hIID and $\alpha \beta+\gamma$ contribute to stimulation of promoter binding by Zta. $\alpha \beta+\gamma$ did not significantly effect the binding of $Z$ ta in the absence of hIID (lane 8). These results confirm previous observations (Lieberman and Berk 1994) that Zta stimulates the formation of an hIID-TFIIA promoter complex and clearly demonstrate that the $\alpha \beta+\gamma$ polypeptides are the source of this function in the DE-IIA fraction (Cortes et al. 1992).

The Zta-dependent stimulation of the hIID-TFIIA complex was analyzed further by $\mathrm{Mg}$-agarose EMSA, which emphasizes the stabilization effect of the formed complex (Fig. 6B). Under limiting conditions for hIID 
A

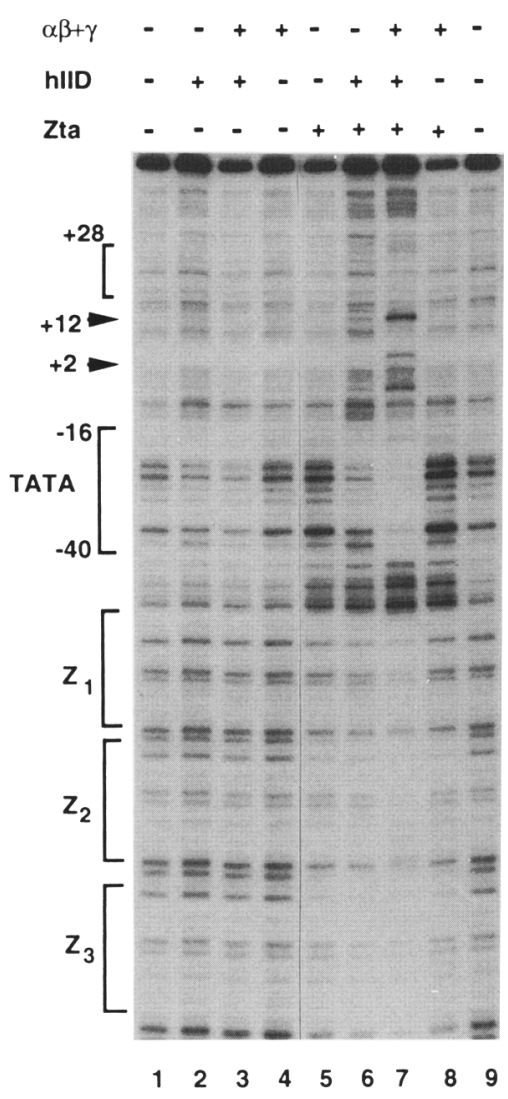

B

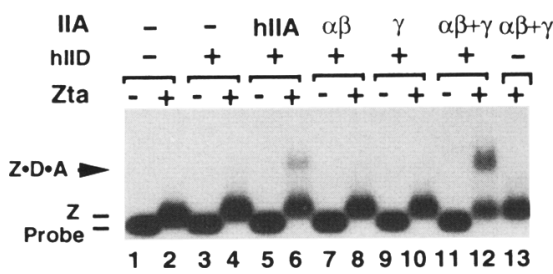

Figure 6. Recombinant TFIIA mediates activator stimulation of TFIID-promoter binding. (A) DNase I footprinting analysis of the $\mathrm{Zta}$-TFIID-TFIIA complex formed on the $\mathrm{Z}_{7} \mathrm{E} 4 \mathrm{~T}$ promoter. TFIIA $(\alpha \beta+\gamma)(0.2 \mu \mathrm{M}), 10 \mu \mathrm{l}$ of immunopurified holo-TFIID (hIID; 2 transcription units), and $12.5 \mathrm{ng}$ of $\mathrm{Zta}$ were incubated with $\sim 6$ fmoles of the promoter DNA for $50 \mathrm{~min}$ prior to DNase I digestion. $(B) \mathrm{Mg}$-agarose EMSA analysis of the Zta-TFIIDTFIIA promoter complex. HeLa cell-derived TFIIA DEAE fraction (hIIA) $(0.75 \mu \mathrm{g})$, or $0.1 \mu \mathrm{M}$ of the recombinant $\alpha \beta, \gamma$, or $\alpha \beta+\gamma$ proteins were incubated with $1 \mu$ l of holo-TFIID (hIID; 0.2 transcription units) and $12.5 \mathrm{ng}$ of $\mathrm{Zta}$ as indicated above each lane. Reactions were incubated at $25^{\circ} \mathrm{C}$ for $30 \mathrm{~min}$.

binding, we found that hIID failed to form a stable EMSA complex by itself (Fig. 6B, lane 3) or in the presence of Zta (lane 4). Addition of partially purified TFIIA (hIIA) also had no effect on hIID binding under these conditions (lane 5). However, in the presence of Zta and hIIA, hIID formed a stable complex (Z-D-A) (lane 6). When the hIIA fraction was substituted with the $\alpha \beta$ polypeptide alone (lanes 7,8 ) or the $\gamma$ polypeptide alone (lanes 9,10), no Z-D-A complex was formed. However, addition of $\alpha \beta+\gamma$ to hIID and $\mathrm{Zta}$ resulted in a strong stimulation of the Z-D-A complex (lane 12). $\alpha \beta+\gamma$ did not stimulate hIID binding in the absence of Zta (lane 11); $\alpha \beta+\gamma$ and Zta did not form a slow mobility complex in the absence of hIID (lane 13). These results show that recombinant TFIIA $\alpha \beta+\gamma$ acts to mediate the stimulatory effect of Zta on the stable binding of hIID to promoter DNA.

\section{TFIIA interacts with the activation domain of Zta}

Heteromeric TFIIA binds directly to TBP, but it has not been reported yet which subunit of TFIIA makes the predominant contact with TBP (Buratowski et al. 1989; Usuda et al. 1991; Cortes et al. 1992). Radiolabeled $\alpha \beta, \gamma$, or $\alpha \beta+\gamma$ proteins were tested for their ability to interact with glutathione $S$-transferase (GST) or GST-TBP fusion proteins immobilized on glutathione-Sepharose (Fig. 7A). We observed that the $\gamma$-subunit bound weakly but specifically to GST-TBP (lane 3), as did the $\alpha \beta$-subunit (lane 6). Significantly, the combination of the $\alpha \beta+\gamma$-subunits together markedly increased the binding to GSTTBP (lane 9). Radiolabeled $\alpha \beta$ and $\gamma$ did not bind to GST alone (lanes 2,5,8). The radiolabeled luciferase control protein (T3) did not bind to either GST or GST-TBP (lanes 11,12). Although $\alpha \beta$ and $\gamma$ were both capable of making direct contact with TBP, the heterodimer clearly bound with higher affinity.

The interaction of TFIIA subunits with one another was also examined by the GST-fusion protein-binding assay (Fig. 7B). Radiolabeled $\gamma, \alpha \beta$, or luciferase control (Luc) was incubated with GST- $\gamma$, GST- $\alpha \beta$, or GST alone (Fig. 7B). As expected, the $\alpha \beta$-subunit bound to GST- $\gamma$ (lane 5, middle panel), and the $\gamma$-subunit bound to GST$\alpha \beta$ (lane 6, top panel). We also observed that $\gamma$ bound strongly to GST- $\gamma$ (lane 5 , top panel), whereas $\alpha \beta$ bound very weakly to GST- $\alpha \beta$ (lane 6, middle panel), suggesting that a homotypic association of the $\gamma$-subunit contributes to the oligomerization state of TFIIA.

A predicted feature of a transcriptional coactivator is that it mediates the binding of an activator to the general transcriptional machinery (Pugh and Tjian 1992). To determine whether TFIIA binds directly to the Z ta transcriptional activator we assayed ${ }^{35}$ S-labeled $\alpha \beta$ or $\gamma$ in a binding reaction with GST-Zta fusion proteins (Fig. 7B). The amino-terminal fusion of GST to Zta did not disrupt transcriptional activation by GST-Zta in vitro, thus obviating the concern that the GST fusion polypeptide interferes with functional Zta activation domain interactions (data not shown). We found that ${ }^{35}$ S-labeled $\gamma$ specifically bound to GST-Zta (Fig. 7B, lane 3, top panel). The $\gamma$ subunit did not bind to GST alone (lane 2, top panel) nor to a deletion of $\mathrm{Zta}(\Delta \mathrm{Zta})$ that lacks the amino-terminal activation domain (Lieberman and Berk 1994) (lane 4, top panel). This indicates that the interaction of $\gamma$ with Zta is activation domain dependent and further supports the significance of our functional observations (Figs. 2-6). We also observed that ${ }^{35}$ S-labeled $\alpha \beta$ bound weakly to GST-Zta (lane 3, middle panel) but did 
A

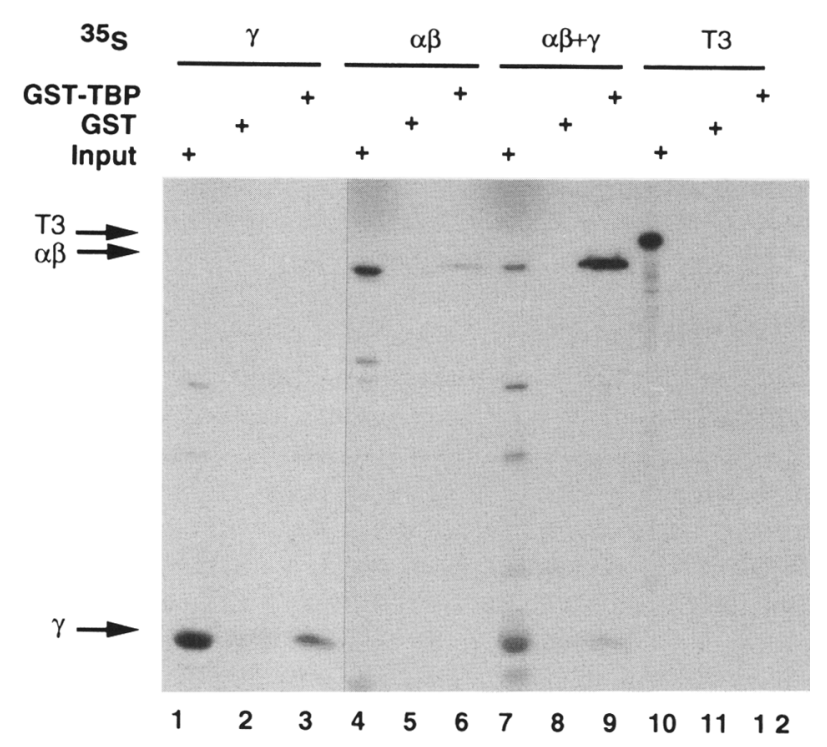

B

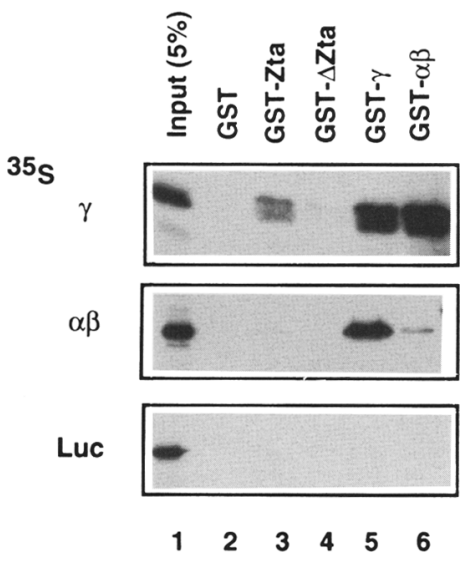

Figure 7. (A) Interaction of TFIIA subunits with TBP. ${ }^{35} \mathrm{~S}-\mathrm{La}$ beled TFIIA $\gamma, \alpha \beta, \alpha \beta+\gamma$, or T3-expressed luciferase control was incubated with GST (lanes 2,5,8,11) or GST-TBP (lanes 3,6,9,12) as indicated above each lane. Input lanes represent $\sim 5 \%$ of the reaction input. ${ }^{35} \mathrm{~S}$-Labeled bound proteins were eluted and analyzed on $12 \%$ SDS-PAGE. (B) TFIIA subunits bind to $\mathrm{Zta}$ in an activation domain-dependent manner. Purified GST fusion proteins of Zta, $\Delta \mathrm{Zta}_{\mathrm{ta}}$, TFIIA $\alpha \beta$ or $\gamma$ (indicated above) were incubated with in vitro-translated ${ }^{35}$ S-labeled $\gamma$ (top panel), $\alpha \beta$ (middle panell, or the luciferase control (Luc; bottom panel). (Lane 1) $5 \%$ of the input labeled protein.

not bind to GST- $\Delta$ Zta (lane 4, middle panel) or GST alone (lane 2, middle panel). Surprisingly, the combination of radiolabeled $\alpha \beta$ and $\gamma$ did not enhance the binding to GST-Zta, as might be expected from additive interactions of the individual subunits with $Z$ ta /data not shown $\mid{ }^{35} \mathrm{~S}$-Labeled luciferase did not bind to any of the GST fusion proteins, indicating that these interactions are specific. These results support the model predicting that the activation domain of $\mathrm{Zta}$ directly targets a surface of TFIIA.
TFIIA and TAFs mediate TATA independent TFIID binding

TAFs have been shown to interact with promoter sequences downstream from the TATA element (Zhou et al. 1992, Kaufman and Smale 1994; Purnell et al. 1994; Verrijzer et al. 1994), and TFIIA has been shown to extend the TBP footprint to the -40 region of certain promoters (Buratowski et al. 1989; Maldonado et al. 1990). These interactions may result in TFIID binding that is resistant to competition from a TATA sequence alone. To test this possibility and to determine the role of TFIIA and the TAFs in the formation of a stable TFIIDpromoter complex, a 200 -bp promoter probe $\left(\mathrm{Z}_{7} \mathrm{E} 4 \mathrm{~T}\right)$ was incubated with factors in the presence or absence of a 100-fold molar excess of cold oligonucleotide containing the E1B TATA element. Incubation of promoter probe with immunopurified hIID $(2 \mu 1)$ produced an EMSA complex in the absence of TFIIA or Zta (Fig. 8, lane 1). Addition of TATA oligonucleotide to the binding reaction with hIID alone resulted in a complete loss of bound complex (Fig. 8, lane 2). Addition of $\alpha \beta+\gamma$ had a weak stimulatory effect on hIID binding (lane 3) (about threefold) but increased the relative amounts of the D-A complex that was resistant to oligonucleotide challenge (lane 4). Zta also stimulated the amount of complex formed (lane 5) but had no effect on the formation of a TATAresistant complex (lane 6). The combination of $Z$ ta and $\alpha \beta+\gamma$ stimulated hIID binding $\sim 10$-fold (lane 7) and produced a complex that was $90 \%$ resistant to TATA oligonucleotide competition (lane 8 ). The importance of the TAFs in the formation of this resistant complex was investigated by replacing hIID with $\mathrm{r}$-huTBP. Addition of $50 \mathrm{ng}$ of hu-rTBP in the presence of $\alpha \beta+\gamma$ and Zta resulted in nearly a complete shifting of the promoter DNA (lane 9). Addition of TATA oligonucleotide resulted in complete disruption of this complex (lane 10). Complexes formed with $\mathrm{r}$-huTBP were highly sensitive to the addition of TATA oligonucleotide, regardless of the presence of $\alpha \beta+\gamma$ (lanes 11,12) or Zta (lanes 13,14). These experiments demonstrate that TFIIA and TAFs augment the binding properties of TBP by establishing a TATA oligonucleotide-resistant complex and this property is significantly stimulated by a promoter specific activator, like Zta.

\section{Discussion}

Previous studies revealed that TFIIA plays an important role in early events in transcriptional activation (Wang et al. 1992; Chi and Carey 1993; Lieberman and Berk 1994). In an effort to understand these early events better, we have isolated a cDNA for the small $(\gamma)$ subunit of human TFIIA. This report shows that TFIIA is an essential component of the general transcriptional machinery required for response to activators. We have found that recombinant TFIIA was required for transcriptional activation from several distinct activation domains in HeLa cell nuclear extracts specifically depleted of TFIIA (Fig. 3), as well as in transcription reactions reconstituted with im- 


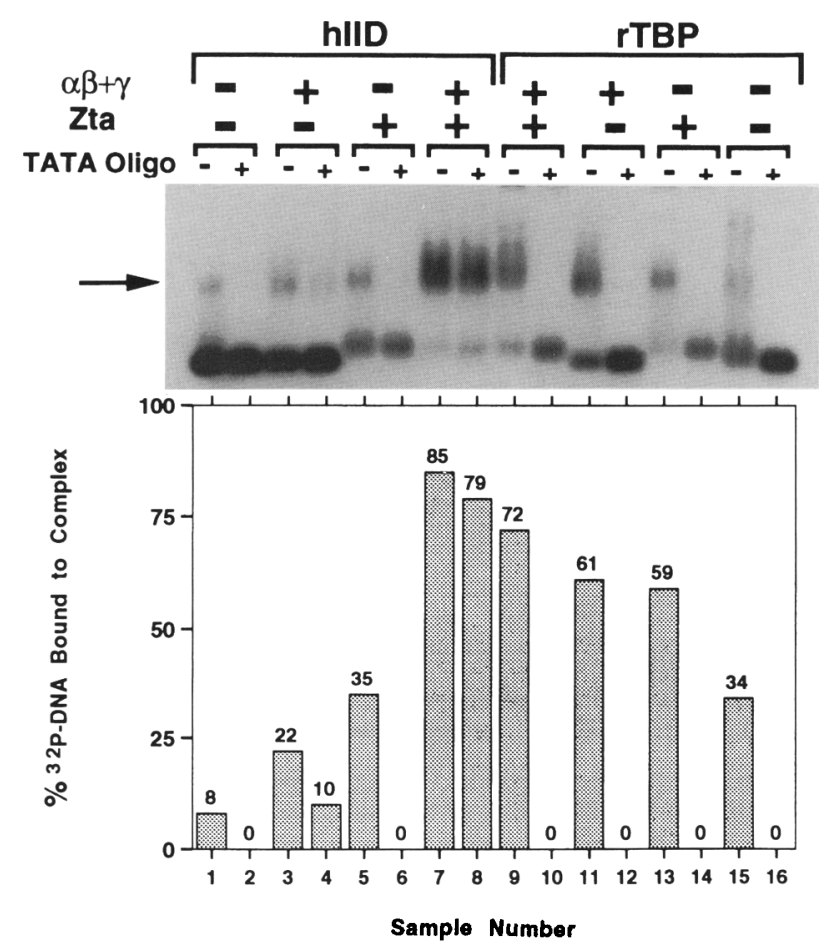

Figure 8. Role of TFIIA and TAFs in TATA oligonucleotide refractory complexes measured by $\mathrm{Mg}$-agarose EMSA. hIID $/ 2$ $\mu l_{i}$ lanes 1-8) or human $\mathrm{rTBP}(50 \mathrm{ng}$; lanes 9-16) was incubated with $0.2 \mu M \alpha \beta+\gamma$ (lanes 3,4,7-12), or Zta (lanes 5-10,13,14) for $45 \mathrm{~min}$ at $30^{\circ} \mathrm{C}$. Unlabeled TATA oligonucleotide was included in even-numbered lanes at a final concentration of $0.04 \mu \mathrm{M} / 100$ fold molar excess|. Quantitation of percent bound complexes was determined by PhosphorImager analysis (Molecular Dynamics).

munopurified TFIID and partially purified HeLa cell transcription factors (Figs. 4 and 5). At least for the $\mathrm{Zta}$ transcriptional activator, TFIIA is likely to be a principal binding target. An interaction of Zta with TFIID is strongly augmented by the addition of TFIIA (Fig. 6) and significantly, TFIIA bound specifically to Zta in an activation domain-dependent manner (Fig. 7). Taken together, these results support a model in which TFIIA mediates the interaction of an activator with TFIID.

\section{Evolutionary conservation of TFIIA function}

The small subunit of hIIA was identified based upon its sequence similarity to yeast TOA2. The functional interchangeability of the yeast and human $\gamma$-subunits in in vitro transcription reactions and TBP-binding assays (Fig. 5) suggests that TFIIA function is conserved between yeast and human. We have shown that TFIIA is essential for transcriptional activation in human in vitro transcription reactions (Figs. 3 and 4). Because both yeast TFIIA subunits are essential for viability (Ranish et al. 1992), a logical prediction is that TFIIA is essential for transcriptional activation in yeast. However, cell-free transcription systems derived from yeast do not require TFIIA activity either for basal or activated transcription (Kim et al. 1994; Koleske and Young 1994). Yeast RNA polymerase II can be isolated as a holoenzyme associated with multiple polypeptides that mediate transcriptional activation when supplemented with TBP and TFIIE (Koleske and Young 1994). These mediators do not appear to be homologs of TAFs nor do they contain TFIIA. This yeast in vitro transcription system appears to be an activation pathway that is distinct from the TAF-dependent system identified in higher eukaryotes. TAF- and TFIIA-independent activation may be the primary mode of regulated transcription in yeast. Alternatively, it is possible that several distinct activation pathways exist in eukaryotes. This latter possibility is supported by the finding of essential yeast genes with sequence similarity to the TAFs (Poon and Weil 1993; Verriizer et al. 1994) and data presented in this report (Fig. 5) demonstrating that yTFIIA supports Zta-activated transcription in a human system.

\section{Role of TFIIA in regulated transcription}

TFIIA has been postulated to prevent inhibitors of TBP from repressing transcription (Roeder 1991; Cortes et al. 1992). Several repressors have been described that directly inhibit TBP function. For example, $\mathrm{Dr}_{1}$ represses transcription by binding directly to TBP and blocking preinitiation complex formation (Inostroza et al. 1992). Similarly, the ATP-dependent inhibitor (ADI) represses transcription by preventing TBP binding to DNA /Auble and Hahn 1993). The binding of TFIIA to TBP may help to preclude these repressors from inactivating TBP. Our results do not rule out a model in which TFIIA functions as a derepressor of TFIID. In our reconstituted transcription reactions, a USA-like coactivator fraction is required to obtain high levels of activated transcription. Transcriptional coactivators, like USA and $\mathrm{Dr}_{2}$, possess both stimulatory activity in the presence of an activator and a repressing activity under basal conditions (Meisterernst et al. 1991; Merino et al. 1993). General repressors of basal transcription, like histone $\mathrm{Hl}$ and core histones, also lead to high levels of induced transcription by promoter-specific activators (Croston et al. 1991; Workman et al. 1991). It is possible that activators, like $\mathrm{Zta}$, stimulate the formation of an hIID-TFIIA promoter complex that is refractory to transcriptional repression.

While repression may be an integral part of the activation mechanism, evidence presented here is also consistent with a model in which TFIIA functions as a positive coactivator. Recombinant TFIIA was shown to interact directly with TBP and the Zta activator (Fig. 7), thus serving as a molecular bridge between an activator and the basal transcriptional machinery. Previous results with partially purified TFIIA revealed (Lieberman and Berk 1994), and those presented here with recombinant TFIIA confirm, that TFIIA augments TFIID binding to promoter DNA in an activator-dependent manner (Fig. $6 \mathrm{~A}, \mathrm{~B})$, thus stimulating the first step in preinitiation complex formation. Furthermore, TFIIA facilitated 
TFIID interactions with downstream promoter sequences in the presence of $\mathrm{Zta}$ (Fig. 6A). Interaction of TFIID with the sequences overlapping the promoter start site has been correlated with transcriptional activity (Kaufman and Smale 1994; Purnell et al. 1994). We have also observed that TFIIA and TAFs function in association with the Zta activator to form a TATA oligonucleotide-resistant TBP-promoter complex (Fig. 8). This Z-D-A complex was refractory to 100 -fold molar excess of TATA oligonucleotide, and this stability is likely a result of the network of protein-protein and proteinDNA interactions that surround the TBP-TATA contact (Lieberman and Berk 1994). Thus, TFIIA is an integral component of an activator-dependent stable preinitiation complex committed to transcription. This form of promoter selectivity may be important for the activation of specific transcripts in the context of the cellular genome, where a vast excess of competing TATA sequences may be accessible.

\section{Is TFIIA a regulatable TAF?}

TAFs mediate the interaction of TBP with several transcriptional activator proteins by a combination of direct and indirect protein interactions /Goodrich et al. 1993; Hoey et al. 1993; Ruppert et al. 1993; Weinzierl et al. 1993; Kokubo et al. 1994). Similarly, we have shown that TFIIA mediates the interaction of TFIID with the Zta transcriptional activator. TAFs have been shown to be essential for activated transcription from almost all activators examined (Pugh and Tjian 1992; Dynlacht et al. 1991; Tanese et al. 1991; Zhou et al. 1992). Similarly, we have shown that TFIIA is required for transcription regulated by several distinct classes of activators. Both TFIIA and TAFs have no clear function in transcription reactions reconstituted with highly purified general factors and the TBP subunit of TFID (Cortes et al. 1992; Tyree et al. 1993). The hallmark feature of TAFs is their tight association with TBP (Dynlacht et al. 1991; Tanese et al. 1991). TFIIA binds TBP with an affinity approaching that of the TAFs, and in Drosophila embryo extracts, the TFIIA large subunit was isolated as a TAF during immunopurification of TBP (Yokomori et al. 1993). Thus, in mammalian systems, TFIIA may be considered a dissociable TAF complex. The relatively weak binding of TFIIA to TBP alone provides a potentially important aspect of biochemical regulation that is distinct from the irreversibly bound TAFs. The tendency toward dissociation of TFIIA from TFIID allows activators to facilitate a reassociation reaction, which may be a rate-limiting step in preinitiation complex assembly (Wang et al. 1992).

The isolation of the cDNA encoding human TFIIA- $\gamma$ has allowed us to report that recombinant TFIIA functions are important for activated transcription. Recombinant TFIIA was found to bind to both TBP and the Zta activator and is likely to function as a stabilizer of this multiprotein complex bound to DNA. This is the first report of an activator making direct contact with the GTF TFIIA, thus providing further evidence that activators contact multiple surfaces of the preinitiation com- plex. The conservation of TFIIA sequence and function between humans and yeast is likely to reflect the importance of TFIIA in fundamental aspects of regulated eukaryotic transcription.

\section{Materials and methods}

\section{Plasmid constructs}

The E. coli expression construct for human TFIIA- $\gamma$ (pQIIA- $\gamma$ ) was derived by PCR amplification of the TFIIA- $\gamma$ cDNA with a BamHI restriction site immediately preceding the amino-terminal ATG initiation codon and a HindIII restriction site immediately following the carboxy-terminal termination codon. This fragment was cloned into the BamHI and HindIII site of pQE-9 (QIAGEN). A similar strategy was used to design the TFIIA- $\alpha \beta$, TFIIA- $\alpha$, and TFIIA- $\beta$ expression constructs, pQIIA- $\alpha \beta$, pQIIA$\alpha$, and pQIIA- $\beta$, respectively. GST- $\gamma$ was derived from the expression plasmid pGST-IIA- $\gamma$, which was constructed by inserting the BamHI-HindIII (filled-in) fragment from pQIIA- $\gamma$ into the BamHI-EcoRI (filled-in) sites of pGEX-2T (Pharmacia). pGST-IIA- $\alpha \beta$ was obtained as a gift from D. Reinberg (Ma et al. 1993). pGST-TBP was a gift from X. Liu and A. Berk. pGST-Zta and pGST- $\Delta Z$ ta were made by inserting the $Z$ ta or $\Delta Z$ ta $(\Delta 2$ 140 ) cDNA (derived from $\mathrm{pH}_{6} \mathrm{Zta}$ or $\mathrm{pH}_{6} \Delta \mathrm{Zta}$, respectively) (Lieberman and Berk 1994) into the BamHI site of PGEX-2T.

\section{Isolation of a cDNA encoding the human TFIIA- $\gamma$ subunit}

The TFIIA- $\gamma$ cDNA was obtained by searching a data base of human cDNA sequence tags obtained at The Institute of Genomic Research and at Human Genome Sciences, Inc., using the BLAST Network Service provided by NCBI. Several ESTs demonstrating homology to TOA2 were identified, including a fulllength cDNA derived from a human T-cell library. This clone was completely sequenced and used for further studies. A stop codon exists just upstream of the initiating codon of the open reading frame with similarity to TOA2. Gaps $(-)$ were introduced in Figure 1 to obtain maximum similarity between yeast and human TFIIA- $\gamma$ subunits and were not included in the percent identity calculations. The human TFIIA- $\gamma$ subunit has a translation initiation site $\left(5^{\prime}\right.$-AAGCCAUGG- $\left.3^{\prime}\right)$ that is consistent with the optimal Kozak consensus sequence for translation initiation $\left(5^{\prime}-\mathrm{CC}^{\mathrm{A}} / \mathrm{G} C A U G G-3^{\prime}\right)$. The GenBank accession number for the human TFIIA- $\gamma$ cDNA sequence is U14193.

\section{Protein preparations}

The TFIIA- $\alpha \beta, \alpha, \beta$, and $\gamma$ subunits were expressed in E. coli with six histidine residues fused to the amino terminus. Fusion proteins were isolated under denaturing conditions, purified on Ni-NTA agarose (Qiagen), and renatured by themselves or in stoichiometric combinations by dialysis first against D100 buffer [ $20 \mathrm{~mm}$ HEPES at $\mathrm{pH} 7.9$ (KOH], $20 \%$ glycerol, $0.2 \mathrm{~mm}$ EDTA Na ${ }^{2+}, 100 \mathrm{mM} \mathrm{KCl}, 7 \mathrm{mM} \beta-\mathrm{ME}, 1 \mathrm{~mm}$ PMSF] containing $500 \mathrm{mM}$ urea for $6 \mathrm{hr}$ at $4^{\circ} \mathrm{C}$, with two subsequent changes of D100 buffer every $6 \mathrm{hr}$ at $4^{\circ} \mathrm{C}$. Dialyzed rTFIIA was cleared of precipitates and frozen in small aliquots, as rTFIIA is labile with multiple freeze/thaw cycles. Recombinant TFIIA polypeptides were used at a final concentration of $\sim 0.2 \mu \mathrm{M}$ for transcription reactions and DNA-binding experiments, unless otherwise noted. General transcription factors and Zta were prepared as described previously by Lieberman and Berk (1994). Yeast TFIIA- $\alpha \beta$ and TFIIA- $\gamma$ were purified as described by Ranish et al. (1992). To produce $h \alpha \beta / y \gamma$, TOA2 was purified from SDSPAGE, eluted, mixed with h $\alpha \beta$ in stoichiometric combinations, and dialyzed as described above. GAL4 fusion proteins were 
purified as described by Chasman et al. (1989) and Kim and Roeder (1993).

\section{DNA-binding reactions}

Mg-agarose EMSA was performed as described by Lieberman and Berk (1994). Limiting conditions for Mg-agarose EMSA (Fig. $6 \mathrm{~B})$ involved a fourfold decrease in hIID concentration $(1.0 \mu \mathrm{l})$ and a twofold decrease in TFIIA concentration $(0.1 \mu \mathrm{M})$ and incubation at room temperature, rather than at $30^{\circ} \mathrm{C}$. DNase I footprinting was performed as described by Lieberman and Berk (1994). Acrylamide EMSA conditions for D-A complex formation have been described previously by Kao et al. (1990).

\section{In vitro transcription reactions}

The promoter templates TATA/Inr SP6 (Smale and Baltimore 1989), Z ${ }_{7}$ E4TCAT (Carey et al. 1992), G ${ }_{5}$ E1BTCAT (Lin et al. 1988), pCol-75/ + 63CAT (AP-1), and pCol-60/+63CAT (mt) (Kerppola et al. 1993) were described previously. In vitro transcription reactions contained $100 \mathrm{ng}$ of DNA template, $\sim 100-$ $200 \mathrm{ng}$ of activator protein, and $40 \mu \mathrm{g}$ of nuclear extract in a $50-\mu \mathrm{l}$ final reaction volume incubated for $1 \mathrm{hr}$ at $30^{\circ} \mathrm{C}$. Primer extension reactions were described previously by Zhou et al. (1992). The TFIIA-depleted nuclear extracts used in Figure 3 were prepared by dialyzing HeLa cell nuclear extract in buffer $\mathrm{D} 100$, adding $\mathrm{KCl}$ to a $500 \mathrm{~mm}$ final concentration, followed by incubation with Ni-NTA-agarose beads (Qiagen) ( $150 \mu$ l packed beads $/ 1 \mathrm{mg}$ of nuclear extract) for $35 \mathrm{~min}$ at $4^{\circ} \mathrm{C}$ while rotating. Ni-NTA beads were cleared from the extract and TFIIA-depleted extracts were dialyzed into D100. The extract was cleared of precipitates, frozen on dry ice, and stored in small volumes at $-80^{\circ} \mathrm{C}$. The HeLa cell nuclear extracts used in Figure $2 \mathrm{~B}$ were made as indicated above, except TFIIA was depleted in $750 \mathrm{mM} \mathrm{KCl}$ (final concentration).

\section{GST fusion-binding assay}

Purified GST or GST fusion proteins (400 ng) were incubated with $2 \times 10^{4} \mathrm{cpm}$ of ${ }^{35} \mathrm{~S}$-labeled protein generated from a rabbit reticulocyte coupled transcription/translation system (Promega) in a $20-\mu \mathrm{l}$ reaction volume for $1 \mathrm{hr}$ at $30^{\circ} \mathrm{C}$. Reactions were then incubated with $20 \mu \mathrm{l}$ of glutathione-Sepharose-4B beads in $300 \mu$ l of protein-binding buffer (PBB). PBB contained $20 \mathrm{~mm}$ HEPES (pH 7.9), 20\% glycerol, $0.5 \mathrm{~mm}$ EDTA, $\mathrm{Na}^{2+} / 60$ $\mathrm{mm} \mathrm{KCl}, 6 \mathrm{~mm} \mathrm{MgCl}_{2}, 0.1 \%$ NP-40, 5 mM $\beta$-mercaptoethanol, and $1 \mathrm{mM}$ PMSF. After $1 \mathrm{hr}$ of rotating at $25^{\circ} \mathrm{C}$, the beads were washed four times with $1.5 \mathrm{ml}$ of $\mathrm{PBB}$, and labeled proteins were eluted by boiling in Laemmli loading buffer. Samples were analyzed on $12 \%$ SDS-polyacrylamide gels, enhanced with NaSalycilate, and visualized by autoradiography.

\section{Acknowledgments}

We thank D. Reinberg for providing the TFIIA- $\alpha \beta$ cDNA, S. Hahn for providing the yeast TOA 1 and TOA2 expression vectors, T. Curran for providing the collagenase gene templates, and R.G. Roeder for providing the Gal4-CTF(399-499) expression construct. We thank Dr. P. Familletti for assistance with large-scale preparation of cell culture and Tina Bryne for preparation of this manuscript. The contribution of the sequencing facility at Human Genome Sciences is acknowledged. Sequence information on one of the TFIIA $\gamma$ ESTs was provided by the Institute of Genomic Research. Human tissue for the construction of several cDNA libraries was provided by Cooperative Human Tissue Network, an agency funded by the National Cancer Institute. P.A.M. is supported by an American Foundation for AIDS Research Scholarship.
The publication costs of this article were defrayed in part by payment of page charges. This article must therefore be hereby marked "advertisement" in accordance with 18 USC section 1734 solely to indicate this fact.

\section{References}

Adams, M.D., J.M. Kelley, J.D. Gocayne, M. Dubnick, M.H. Polymeropoulos, H. Xiao, C.R. Merril, A. Wu, B. Olde, and R.F. Moreno, A.R. Kerlavage, W.R. McCombie, and J.C. Venter. 1991. Complementary DNA sequencing: Expressed sequence tags and human genome project. Science 252: 16511656.

Adams, M.D., M. Dubnick, A.R. Kerlavage, R. Moreno, J.M. Kelley, T.R. Utterback, J.W. Nagle, C. Fields, and J.C. Venter. 1992. Sequence identification of 2,375 human brain genes. Nature 355: 632-634.

Altschul, S.F., W. Gish, W. Miller, E.W. Myers, and D.J. Lipman. 1990. Basic local alignment search tool. I. Mol. Biol. 215: 403-410.

Auble, D.T and S. Hahn. 1993. An ATP-dependent inhibitor of TBP binding to DNA. Genes \& Dev. 7: 844-856.

Buratowski, S. and H. Zhou. 1992. Transcription factor IID mutants defective for interaction with transcription factor IIA. Science 255: 1130-1132.

Buratowski, S., S. Hahn, L. Guarente, and P.A. Sharp. 1989. Five intermediate complexes in transcription initiation by RNA polymerase II. Cell 56: 549-561.

Carey, M., J. Kolman, D.A. Katz, L. Gradoville, L. Barberis, and G. Miller. 1992. Transcriptional synergy by the Epstein-Barr virus transactivator ZEBRA. I. Virol. 66: 4803-4813.

Chasman, D.I., J. Leatherwood, M. Carey, M. Ptashne, and R.D. Kornberg. 1989. Activation of yeast polymerase II transcription by herpes virus VP16 and GAL4 derivatives in vitro. Mol. Cell. Biol. 9: 4746-4749.

Chi, T. and M. Carey. 1993. The ZEBRA activation domain: Modular organization and mechanism of action. Mol. Cell. Biol. 13: 7045-7055.

Conaway, R.C. and J.W. Conaway. 1993. General initiation factors for RNA polymerase II. Annu. Rev. Biochem. 62: 161190.

Cortes, P., O. Flores, and D. Reinberg. 1992. Factors involved in specific transcription by mammalian RNA polymerase II: Purification and analysis of transcription factor IIA and identification of transcription factor III. Mol. Cell. Biol. 12: 413421.

Croston, G.E., L.A. Kerrigan, L.M. Lira, D.R. Marshak, and J.T. Kadonaga. 1991. Sequence-specific antirepression of histone H1-mediated inhibition of basal RNA polymerase II transcription. Science 251: 643-649.

DeJong, J. and R.G. Roeder. 1993. A single cDNA, hTFIIA/ $\alpha$, encodes both the p35 and p19 subunits of human TFIIA. Genes \& Dev. 7: 2220-2234.

Drapkin, R., A. Merino, and D. Reinberg. 1993. Regulation of RNA polymerase II transcription. Curr. Opin. Cell. Biol. 5: $469-476$.

Dynlacht, B.D., T. Hoey, and R. Tjian. 1991. Isolation of coactivators associated with the TATA-binding protein that mediate transcriptional activation. Cell 66: 563-576.

Gill, G. and R. Tiian. 1992. Eukaryotic coactivators associated with the TATA box binding protein. Curr. Opin. Genet. Dev. 2: 236-242.

Goodrich, J.A., T. Hoey, C.J. Thut, A. Admon, and R. Tjian. 1993. Drosophila TAF $_{\mathrm{II}} 40$ interacts with both a VP16 activation domain and the basal transcription factor TFIIB. Cell 
75: 519-530.

Hahn, S., S. Buratowski, P.A. Sharp, and L. Guarente. 1989. Identification of a yeast protein homologous in function to the mammalian general transcription factor, TFIIA. EMBO $J$. 8: 3379-3382.

Hoey, T., R.O.J. Weinzierl, G. Gill, J.-L. Chen, B.D. Dynlacht, and R. Tjian. 1993. Molecular cloning and functional analysis of Drosophila TAF110 reveal properties expected of coactivators. Cell 72: 247-260.

Imbalzano, A.N., K.S. Zaret, and R.E. Kingston. 1994. Transcription factor (TF) IIB and TFIIA can independently increase the affinity of the TATA-binding protein for DNA. $I$. Biol. Chem. 269: 8280-8286.

Inostroza, J.A., F.H. Mermelstein, I. Ha, W.S. Lane, and D. Reinberg. 1992. Drl, a TATA-binding protein-associated phosphoprotein and inhibitor of class II gene transcription. Cell 70: 477-489.

Kao, C.C., P.M. Lieberman, M.C. Schmidt, Q. Zhou, R. Pei, and A.J. Berk. 1990. Cloning of a transcriptionally active human TATA binding factor. Science 248: 1646-1650.

Kaufmann, J. and S.T. Smale. 1994. Direct recognition of initiator elements by a component of the transcription factor IID complex. Genes \& Dev. 8: 821-829.

Kerppola, T.K., D. Luk, and T. Curran. 1993. Fos is a preferential target of glucocorticoid receptor inhibition of AP-1 activity in vitro. Mol. Cell. Biol. 13: 3782-3791.

Kim, T.K. and R.G. Roeder. 1993. Transcriptional activation in yeast by the proline-rich activation domain of human CTF1. I. Biol. Chem. 268: 20866-20869.

Kim, Y.-J., S. Bjorklund, Y. Li, M.H. Sayre, and R.D. Kornberg. 1994. A multiprotein mediator of transcriptional activation and its interaction with the C-terminal repeat domain of RNA polymerase II. Cell 77: 599-608.

Kokubo, T., D.-W. Gong, J.C. Wootton, M. Horikoshi, R.G. Roeder, and Y. Nakatani. 1994. Molecular cloning of Drosophila TFIID subunits. Nature 367: 484-487.

Koleske, A.J. and R.A. Young. 1994. An RNA polymerase II holoenzyme responsive to activators. Nature 368: 466-469.

Lee, D.K., J. DeJong, S. Hashimoto, M. Horikoshi, and R.G. Roeder. 1992. TFIIA induces conformational changes in TFIID via interactions with the basic repeat. Mol. Cell. Biol. 12: 5189-5196.

Lieberman, P.M. and A.J. Berk. 1994. A mechanism for TAFs in transcriptional activation: Activation domain enhancement of TFIID-TFIIA-promoter DNA complex formation. Genes \& Dev. 8: 995-1006.

Lin, Y.-S., M.F. Carey, M. Ptashne, and M.R. Green. 1988. GAL4 derivatives function alone and synergistically with mammalian activators in vitro. Cell 54: 659-664.

Ma, D., H. Watanabe, F. Mermelstein, A. Admon, K. Oguri, X. Sun, T. Wada, T. Imai, T. Shiroya, D. Reinberg, and H. Handa. 1993. Isolation of a cDNA encoding the largest subunit of TFIIA reveals functions important for activated transcription. Genes \& Dev. 7: 2246-2257.

Maldonado, E., I. Ha, P. Cortes, L. Weis, and D. Reinberg. 1990. Factors involved in specific transcription by mammalian RNA polymerase II: Role of transcription factors IIA, IID, and IIB during formation of a transcription-competent complex. Mol. Cell. Biol. 10: 6335-6347.

Meisterernst, M. and R.G. Roeder. 1991. Family of proteins that interact with TFIID and regulate promoter activity. Cell 67: $557-567$.

Meisterernst, M., A.L. Roy, H.M. Lieu, and R.G. Roeder. 1991. Activation of class II gene transcription by regulatory factors is potentiated by a novel activity. Cell 66: 981-993.

Merino, A., K.R. Madden, W.S. Lane, J.J. Champoux, and D.
Reinberg. 1993. DNA topoisomerase I is involved in both repression and activation of transcription. Nature 365: 227232.

Poon, D. and P.A. Weil. 1993. Immunopurification of yeast TATA-binding protein and associated factors. J. Biol. Chem. 268: 15325-15328.

Pugh, B.F. and R. Tjian. 1992. Diverse transcriptional functions of the multisubunit eukaryotic TFIID complex. I. Biol. Chem. 267: 679-682.

Purnell, B.A., P.A. Emanuel, and D.S. Gilmour. 1994. TFIID sequence recognition of the initiator and sequences farther downstream in Drosophila class II genes. Genes \& Dev. 8: $830-842$

Ranish, J.A. and S. Hahn. 1991. The yeast general transcription factor TFIIA is composed of two polypeptide subunits. $I$. Biol. Chem. 266: 19320-19327.

Ranish, J.A., W.S. Lane, and S. Hahn. 1992. Isolation of two genes that encode subunits of the yeast transcription factor IIA. Science 255: 1127-1129.

Roeder, R.G. 1991. The complexities of eukaryotic transcription initiation: Regulation of preinitiation complex assembly. Trends Biochem. Sci. 16: 402-408.

Ruppert, S., E.H. Wang, and R. Tjian. 1993. Cloning and expression of human $\mathrm{TF}_{\mathrm{II}} 250$ : A TBP-associated factor implicated in cell-cycle regulation. Nature 362: 175-179.

Smale, S.T. and D. Baltimore. 1989. The "initiator" as a transcription control element. Cell 57: 103-113.

Tanese, N., B.F. Pugh, and R. Tjian. 1991. Coactivators for a proline-rich activator purified from the multisubunit human TFIID complex. Genes \& Dev. 5: 2212-2224.

Tiian, R. and T. Maniatis. 1994. Transcriptional activation: A complex puzzle with few easy pieces. Cell 77: 5-8.

Tyree, C.M., C.P. George, L.M. Lira-DeVito, S.L. Wampler, M.E. Dahmus, L. Zawel, and J.T. Kadonaga. 1993. Identification of a minimal set of proteins that is sufficient for accurate initiation of transcription by RNA polymerase II. Genes \& Dev. 7: 1254-1265.

Usuda, Y., A. Kubota, A. Berk, and H. Handa. 1991. Affinity purification of transcription factor IIA from Hela cell nuclear extracts. EMBO I. 10: 2305-2310.

Verrijzer, C.P., K. Yokomori, J.-L. Chen, and R. Tjian. 1994. Drosophila $\mathrm{TAF}_{\mathrm{II}}$ 150: Similarity to yeast gene TSM-1 and specific binding to core promoter DNA. Science 264: 933941.

Wang, W., J.D. Gralla, and M. Carey. 1992. The acidic activator GAL4-AH can stimulate polymerase II transcription by promoting assembly of a closed complex requiring TFIID and TFIIA. Genes \& Dev. 6: 1716-1727.

Weinzierl, R.O.J., S. Ruppert, B.D. Dynlacht, T. Naoko, and R. Tjian. 1993. Cloning and expression of Drosophila TAF $_{\mathrm{II}} 60$ and human $\mathrm{TAF}_{\mathrm{II}} 70$ reveal conserved interactions with other subunits of TFIID. EMBO f. 12: 5303-5309.

Workman, I.L., I.C.A. Taylor, and R.E. Kingston. 1991. Activation domains of stably bound Gal4 derivatives alleviate repression of promoters by nucleosomes. Cell 64: 533-544.

Yokomori, K., A. Admon, J.A. Goodrich, J.-L. Chen, and R. Tiian. 1993. Drosophila TFIIA-L is processed into two subunits that are associated with the TBP/TAF complex. Genes \& Dev. 7: 2235-2245.

Zawel, L. and D. Reinberg. 1993. Initiation of transcription by RNA polymerase II: A multi-step process. Prog. Nucleic Acid Res. Mol. Biol. 44: 67-108.

Zhou, Q., P.M. Lieberman, T.G. Boyer, and A.J. Berk. 1992. Holo-TFIID supports transcriptional stimulation by diverse activators and from a TATA-less promoter. Genes \& Dev. 6: 1964-1974. 


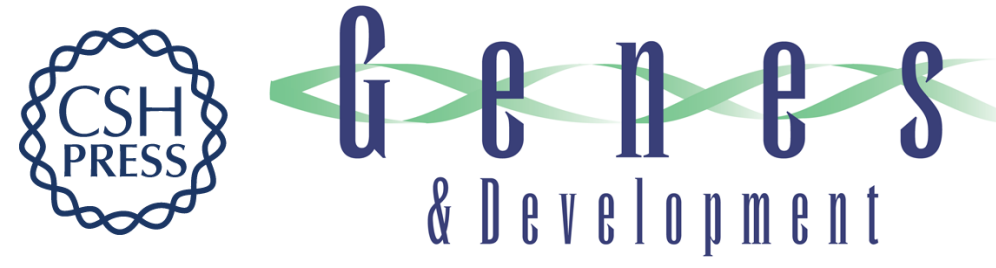

\section{Molecular cloning of the small (gamma) subunit of human TFIIA reveals functions critical for activated transcription.}

J Ozer, P A Moore, A H Bolden, et al.

Genes Dev. 1994, 8:

Access the most recent version at doi:10.1101/gad.8.19.2324

References This article cites 55 articles, 29 of which can be accessed free at:

http://genesdev.cshlp.org/content/8/19/2324.full.html\#ref-list-1

License

Email Alerting

Service

Receive free email alerts when new articles cite this article - sign up in the box at the top right corner of the article or click here.

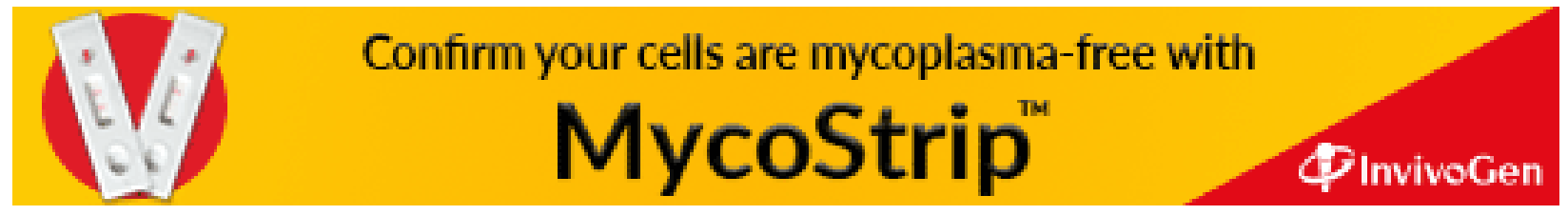

